\title{
The 2019 revision of the International Standards for Neurological Classification of Spinal Cord Injury (ISNCSCI)- What's new?
}

\author{
ASIA and ISCoS International Standards Committee
}

Received: 7 August 2019 / Accepted: 7 August 2019 / Published online: 17 September 2019

(c) International Spinal Cord Society 2019

The International Standards for Neurological Classification of Spinal Cord Injury (ISNCSCI) continuously maintained by the International Standards Committee of the American Spinal Injury Association (ASIA) and the International Spinal Cord Society (ISCoS) represents the gold standard assessment for documentation of the level and severity of a spinal cord injury. Since its first introduction, the ISNCSCI has undergone several revisions [1] with its newest eighth edition [2] released in April this year. The new definitions introduced by the 2019 revision are not only based upon general comments, questions, and suggestions from the international SCI community including clinicians as well as researchers, but also take recently available evidence from ISNCSCI data analyses and structured feedback from ISNCSCI training courses and workshops into account. The main changes include (1) a new taxonomy for systematic documentation of clinical judgment in the presence of nonSCI related conditions, and (2) a new definition of the Zones of Partial Preservation (ZPP), which applies now not only to complete, but also to incomplete injuries with missing motor or sensory function in the lowest sacral segments.

\section{Taxonomy for documentation of non-SCI related conditions}

Based on the feedback from clinicians, there is an increasing number of patients with pre-existing or concomitant musculoskeletal, neurological, or other non-SCI related

Rüdiger Rupp

ruediger.rupp@med.uni-heidelberg.de

1 Spinal Cord Injury Center, Heidelberg University Hospital, Heidelberg, Germany problems, which have an impact on the ISNCSCI examination and classification results. Such problems include chronic peripheral nerve injuries, amputations, fractures, burns, acute or chronic pain or age related or immobility related muscle weaknesses. In a previous ISNCSCI revision, the motor score " 5 *" was intended for such cases, where the full muscle strength was not achieved, but the examiner believed that it would have been achieved if the non-SCI related condition was not present. However, this approach has the drawback that (1) it has been reserved for the motor examination only and (2) the actual examination score is lost unless the examiner explicitly documents it in the comments box of the worksheet.

To overcome this limitation, a general “*”-concept has been introduced, where abnormal examination scores can be tagged with a "*”" to indicate that a non-SCI condition impacts the examination results. This general "**"-concept is applicable to the sensory as well as the motor exam independent from the level of occurrence (above, at or below the sensory/motor level). The " 5 *"-score is not used anymore, instead the actual (not normal) examination score is documented and tagged with the "**". As such for motor strength, the "**" can be applied to motor scores of $0,1,2,3,4$ or for NT (not testable), and this can also be applied to sensory scores of 0,1 or NT. For ongoing clinical studies, where the " 5 *" is currently being used, it is not recommended that changes be made in the documentation. However, as outlined in a recent joint publication of the ASIA and ISCoS International Standards Committee and the ASIA Educational Committee [3] information on the ISNCSCI revision used in the study should be given in publications.

If an examiner tags a score with the "*", details on the reason for this and how to handle this "**"-tagged score during the classification process should be specified in the comments box on the front side of the ISNCSCI worksheet. While "*”-tagged scores above the sensory/motor level will in most cases be handled as normal during classification, 
“*”-tagged scores at or below the motor/sensory level indicating a non-SCI related impairment superimposed to the deficit caused by the SCI will typically be handled as not normal. Each classification variable resulting in defined motor, sensory levels, Neurological Level of Injury (NLI), or ASIA Impairment Scale (AIS) grade which is affected by any "*"-tagged score, should also be designated with a "*". By this method, it is clearly indicated that the classification results are based on clinical interpretation of the actual observed and recorded scores.

\section{Definition of Zones of Partial Preservation (ZPP) in incomplete lesions}

The ZPPs represent important pieces of information for the characterization of the extent of preserved functions below the sensory and motor levels. As such, ZPPs are among the most important predictors of neurological recovery [4]. In the 2011 ISNCSCI revision and the 2015 update, ZPPs were only defined for clinically complete (AISA) injuries with no sensorimotor function in the most caudal sacral segments. Recording ZPPs only in cases with totally lost sensation (absent deep anal pressure (DAP); absent light touch (LT) and pin prick (PP) sensation) in S4-5 and lost sacral motor function (no voluntary anal contraction (VAC)) is not intuitive and restricts the value of ZPPs for effective clinical communication to AIS A lesions only.

Therefore, the ZPP rules were refined and are no longer based on the AIS grade. Motor ZPPs are now defined and should be documented in all cases including patients with incomplete injuries with absent VAC. The sensory ZPP on a given side is defined in the absence of sensory function in S4-5 (LT, PP) on this side as long as DAP is not present. This means that in cases with present DAP, sensory ZPPs on both sides are not defined and should be noted as "not applicable (NA)". In cases with absent DAP, a sensory ZPP can be defined on one side (assuming also absent LT and PP sensation in S4-5 on this side), while it may not necessarily be applicable (and should be noted as "NA") on the other side if there is present LT or PP at S4-5.

It has to be emphasized that in clinically complete injuries (AIS A) the new ZPP definition is fully compatible with the former definition and does not lead to different classification results.

An analysis of ISNCSCI datasets from the Spinal Cord Injury Model Systems database [5] and from the European Multicenter Study about Spinal Cord Injury (EMSCI) database found that in one-third of all incomplete patients meaningful ZPPs can be provided with the new definition. A deeper analysis of the EMSCI dataset revealed that the prognosis of the lower extremity motor score after one year is more reliable with the new ZPP definition [6].
The changes introduced by the 8th ISNCSCI edition have already been integrated into the new booklet and worksheet (freely downloadable from both ASIA and ISCoS websites). The implementation of the new definitions into the online training tool InSTeP is to be finalized soon. A current effort of the ASIA and ISCoS International Standards Committee is to publish a series of papers in Spinal Cord summarizing and justifying the changes of the recent revision and providing practical sample cases for better illustration of their correct application. In addition, guidelines for an expedited-ISNCSCI exam (E-ISNCSCI) and additional research options (RO-ISNCSCI) will soon be presented.

In summary, the 2019 ISNCSCI revision represents another milestone in the continuous evolution of the ISNCSCI toward the most inclusive assessment of people with SCI.

Members of the ASIA and ISCoS International Standards Committee R. Betz ${ }^{2}$, F. Biering-Sørensen ${ }^{3}$, S.P. Burns ${ }^{4}$, W. Donovan ${ }^{5}$, D.E. Graves ${ }^{6}$, J. Guest ${ }^{7}$, L. Jones ${ }^{8}$, S. Kirshblum ${ }^{9}$, A. Krassioukov ${ }^{10}$, M.J. Mulcahey ${ }^{6}$, M. Schmidt Read ${ }^{11}$, G.M. Rodriguez ${ }^{12}$, R. Rupp ${ }^{1}$, C. Schuld ${ }^{1}$, K. Tansey ${ }^{13}$, K. Walden ${ }^{14}$

${ }^{2}$ Institute for Spine and Scoliosis, Ocean City, NJ, USA; ${ }^{3}$ Clinic for Spinal Cord Injuries, Rigshospitalet, University of Copenhagen, Copenhagen, Denmark; ${ }^{4}$ Department of Rehabilitation Medicine, University of Washington School of Medicine, Seattle, WA, USA; ${ }^{5}$ Institute for Rehabilitation and Research, Houston, TX, USA; ${ }^{6}$ Thomas Jefferson University, Philadelphia, PA, USA; ${ }^{7}$ Department of Neurological Surgery, University of Miami Miller School of Medicine, Miami, FL, USA; ${ }^{8}$ University of Colorado, Denver, CO, USA; ${ }^{9}$ Kessler Institute for Rehabilitation, Rutgers New Jersey Medical School, NJ, USA; ${ }^{10}$ International Collaboration On Repair Discovery (ICORD), Vancouver, Canada; ${ }^{11}$ Magee Rehabilitation Hospital Jefferson Health, Philadelphia, PA, USA; ${ }^{12}$ University of Michigan, Michigan Medicine, Ann Arbor, MI, USA; ${ }^{13}$ Departments of Neurosurgery and Neurobiology, University of Mississippi Medical Center, Jackson, MS, USA; ${ }^{14}$ Rick Hansen Institute, Vancouver, Canada

\section{Compliance with ethical standards}

Conflict of interest The authors declare that they have no conflict of interest.

Publisher's note Springer Nature remains neutral with regard to jurisdictional claims in published maps and institutional affiliations.

\section{References}

1. Solinsky R, Kirshblum SC. Challenging questions regarding the international standards. J Spinal Cord Med. 2018;41:684-90.

2. American Spinal Injury Association. International standards fo neurological classification of spinal cord injury. Richmond, VA: American Spinal Injury Association; 2019.

3. ASIA and ISCoS International Standards Committee, ASIA Education Committee, Rupp R. Assessor accuracy of the International Standards for Neurological Classification of Spinal Cord Injury 
(ISNCSCI)-recommendations for reporting items. Spinal Cord 2018;56:819-20.

4. Wilson JR, Cadotte DW, Fehlings MG. Clinical predictors of neurological outcome, functional status, and survival after traumatic spinal cord injury: a systematic review. J Neurosurg Spine. 2012;17(1 Suppl):11-26.

5. Kirshblum SC, Botticello AL, Dyson-Hudson TA, Byrne R, Marino RJ, Lammertse DP. Patterns of sacral sparing components on neurologic recovery in newly injured persons with traumatic spinal cord injury. Arch Phys Med Rehabil. 2016;97:1647-55.

6. Schuld C, Franz S, Weidner N, Kirshblum S, Tansey K, Rupp R. Increasing the clinical value of the Zones of Partial PreservationA quantitative comparison of a new definition rule applicable also in incomplete lesions. Topics in Spinal Cord Injury. Top Spinal Cord Injury Rehab. 2018;24(1 Suppl):120-21. 